\title{
Nutrición enteral domiciliaria: descripción de las características clínicas de los pacientes atendidos a través de una consulta telefónica de enfermería
}

\author{
Clinical characteristics of patients with home enteral nutrition receiving \\ telephone nursing support \\ Nutrição enteral domiciliaria: descrição das características clínicas dos pacientes \\ atendidos na consulta telefônica de enfermagem
}

\begin{abstract}
Abel González-González 1*, María Jesús del Hoyo Serrano², Isabel Domínguez Osorio", Belén Fernández de Bobadilla', Carmen Ferreiro Vicario', Pedro Antonio Muñoz Cazallas'.
\end{abstract}

Recibido: 21 de enero 2020. Aceptado para publicación: 29 de marzo 2020

Publicado en línea: 14 de mayo de 2020

https://doi.org/10.35454/rncm.v3n2.58

\section{Resumen}

Introducción: la nutrición enteral domiciliaria (NED) es una alternativa para aquellos pacientes que presentan incapacidad de alimentarse naturalmente a causa de su situación clínica y que no necesitan estar en un medio hospitalario.

Objetivo: describir las características de los pacientes con NED atendidos telefónicamente en una consulta de enfermería y el tipo de complicaciones.

Método: estudio observacional descriptivo transversal de los pacientes con NED. Las variables del estudio incluyeron edad, sexo, vías de acceso, tipo de fórmula nutricional usada y complicaciones.

Resultados: fueron atendidos 148 pacientes, con una media de edad de 65,4 $\pm 14,69$ años; $66,2 \%$ hombres. Las indicaciones más frecuentes de NED fueron: accidentes cerebrovasculares (16,9\%), retraso psicomotor $(10,1 \%)$ y neoplasia de laringe ( $8,1 \%)$. Las fórmulas nutricionales utilizadas fueron normocalóricas $53 \%$, hipercalóricas $47 \%$, normoproteicas $60 \%$, hiperproteicas $40 \%$, con fibra $52 \%$ y $\sin$ fibra $48 \%$. El $68 \%$ de los pacientes tenían un acceso enteral invasivo. El $38 \%$ de ellos presentaron complicaciones, $26 \%$ morta-

\section{Abstract}

Introduction: Home enteral nutrition (HEN) is an alternative for patients who are unable to feed orally because of their clinical situation and who do not need to be hospitalized.

Objective: describe the clinical characteristics and complications patients with HEN receiving telephonic nursing support.

Methods:Observational descriptive study of patients with HEN. Study variables included: age, sex, enteral access, type of nutritional formula used, and type of complications.

Results: A total of 148 patients with HEN were treated. Mean age was $65.4 \pm 14.69$ years and $66.2 \%$ were males. The most common indications of HEN were stroke (16.9\%), psychomotor retardation (10.1\%), and laryngeal cancer (8.1\%). Regarding the type of nutritional formula, $53 \%$ were normocaloric, $47 \%$ hypercaloric, $60 \%$ normoproteic, 40\% hyperproteic, 52\% fiber-enriched, and $48 \%$ were not fiberenriched. Sixty-eight percent of patients had invasive enteral access, and $38.51 \%$ of them developed complications, of which $26.35 \%$ were fatal. Respiratory infections were the main cause of death, and mor-

\section{Resumo}

Introdução: a nutrição enteral domiciliaria (NED) é uma alternativa para os pacientes que não conseguem se alimentar naturalmente, devido à sua situação clínica e que não precisam de estar em ambiente hospitalar.

Objetivo: descrever as características dos pacientes com NED atendidos por telefone em consulta de enfermagem e descrever as suas complicações.

Método: estudo observacional descritivo transversal de pacientes com NED. As variáveis do estudo incluíram idade, sexo, vias de acesso, tipo de fórmula nutricional utilizada e complicações.

Resultados: 148 pacientes foram atendidos, com idade média de 65,4 \pm 14,69 anos; $66,2 \%$ homens e 33,8 \% mulheres. As causas mais frequentes de NED foram: acidente vascular cerebral (16,9\%), retardo psicomotor (10,1\%) e neoplasia da laringe $(8,1 \%)$. As fórmulas nutricionais utilizadas foram: normocalóricas $53 \%$, hipercalóricas $47 \%$, normoproteicas $60 \%$, hiperproteicas $40 \%$, com fibra $52 \%$ e sem fibra $48 \%$. $68 \%$ dos pacientes tiveram acesso enteral invasivo. $38 \%$ apresentaram complicações, $26 \%$ fatais. A principal causa de morte 
les. La principal causa de fallecimiento fue la infección respiratoria. La mortalidad fue más frecuente entre aquellos con acceso enteral invasivo.

Conclusiones: el estudio permitió conocer las características de los pacientes con NED del área de salud perteneciente a la Unidad de Nutrición Clínica del Hospital General Universitario de Ciudad Real en España. La utilización de vías de acceso invasivas es elevada. Los trastornos neurológicos fueron la principal indicación médica para la NED. El papel de enfermería es muy importante en el control ambulatorio de los pacientes con NED.

Palabras clave: nutrición enteral, alimentos formulados, enfermeras. tality was higher in patients with invasive enteral access.

Conclusions: The current study described the clinical characteristics in a sample of patients with HEN from the Clinical Nutrition Unit of the General University Hospital of Ciudad Real (Spain). Use of invasive enteral access in these patients showed to be high. Neurological disease was the main prescription indication. Nursing support is crucial for the outpatient care of patients with HEN.

Keywords: Enteral nutrition; Formulated food; Nurses. foi infeção respiratória. A mortalidade foi mais frequente entre aqueles com acesso enteral invasivo.

Conclusões: o estudo permitiu conhecer as características dos pacientes com NED da área de saúde pertencentes à Unidade de Nutrição Clínica do Hospital Universitário Geral da Cidade Real em Espanha. O uso de rotas de acesso invasivas é alto. Os distúrbios neurológicos foram a principal indicação clínica para NED. O papel da enfermagem é muito importante no controle ambulatório de pacientes com NED.

Palavras-chave: nutrição enteral, alimentos formulados, enfermeiros.
Unidad de Nutrición. Sección de Endocrinología y Nutrición del Hospital General Universitario de Ciudad Real, España.

2 Clínica de Diálisis Nuestra Señora del Prado Ciudad Real. España.
3 Servicio de Medicina Interna del Hospital General Universitario de Ciudad Real, España.

*Correspondencia: Abel González González

abelg@sescam.jccm.es

\section{INTRODUCCIÓN}

El estado nutricional del individuo condiciona su capacidad de respuesta a la enfermedad ${ }^{(1-7)}$. De igual forma, las enfermedades influyen negativamente sobre la situación nutricional porque estas tienen un efecto catabólico y, muchas veces, provocan hipo/anorexia. En ocasiones los pacientes precisan un soporte nutricional especializado (SNE) con el fin de mantener un adecuado estado nutricional.

Los pacientes hospitalizados que necesitan este tipo de SNE, que no tienen otro motivo para mantener el ingreso y que cumplen una serie de criterios respecto al pronóstico o la aceptación de esta terapia por parte de sus cuidadores, pueden volver a su lugar de residencia con nutrición artificial domiciliaria (NAD), preferentemente enteral (NED) o parenteral (NPD; manteniendo un control ambulatorio periódico por la unidad responsable de la indicación o por equipos de atención primaria, debidamente coordinados ${ }^{(8,9)}$.

El carácter impredecible de algunas de las complicaciones asociadas a la NED y su necesidad de resolución inmediata ha llevado a que distintos grupos de expertos y organismos gubernamentales recomienden programas de seguimiento de estos pacientes ${ }^{(3,7,8)}$. Desde la Unidad de Nutrición Clínica del Hospital General Universitario de Ciudad Real (HGUCR) se propuso la creación de una consulta de enfermería específicamente dedicada al seguimiento de los casos de NED, teniendo en cuenta la necesidad de un control estrecho y la demanda de estos pacientes. El objetivo de la consulta fue mantener una comunicación fluida, accesible y permanente con los pacientes con este tipo de tratamiento y con sus familiares/cuidadores con el fin de facilitarles el manejo: resolviendo dudas sobre aspectos clínicos y posibles complicaciones con la sonda, con los dispositivos para la administración de la fórmula pautada o con la fórmula en sí y también con el personal administrativo. Se protocolizó un contacto telefónico mensual para todos los pacientes, aunque esta frecuencia fue mayor según las necesidades de cada uno. Además, el diseño de esta consulta permitió que los pacientes o familiares contactaran motu proprio ante incidencias imprevistas.

El objetivo de este trabajo fue definir las principales características de los pacientes con NED atendidos en la consulta durante los primeros 14 meses de su implementación, así como describir las complicaciones de estos en relación con la terapia. 


\section{MÉTODO}

Se realizó un estudio observacional descriptivo transversal de todos los pacientes con NED atendidos en la consulta de enfermería de nutrición artificial domiciliaria entre el 01/04/2018 y el 01/06/2019 (14 meses).

La consulta de enfermería de NED forma parte de la estructura asistencial de la Unidad de Nutrición Clínica del HGUCR, que es la unidad de referencia de nutrición artificial de nuestra área sanitaria cuya población de referencia es de 263.646 habitantes. Se consideraron pacientes elegibles para el estudio todos aquellos atendidos en la consulta de enfermería de NED, y resultaron excluidos los pacientes que no aceptaron que sus datos fueran utilizados tras ser informados del objetivo del estudio; tal como fue exigido por el Comité Ético de Investigación de la institución.

Se diseñó una base de datos informatizada en la que se recogieron prospectivamente, por parte de los profesionales de enfermería que atienden la consulta (MJHS, CFV y PAMC), las siguientes variables: edad, sexo, vías de acceso de la nutrición enteral, tipo de fórmula nutricional usada y tipo de complicaciones durante el seguimiento. Las complicaciones se clasificaron como debidas a la sonda o a la fórmula nutricional, retirada de la sonda o patología no mortal probablemente asociada al tratamiento. Las vías de acceso se clasificaron en invasivas y no invasivas. Las complicaciones graves fueron aquellas que obligaron a un ingreso hospitalario o las que resultaron en el fallecimiento del paciente. Estos datos fueron revisados por los médicos especialistas en Nutrición de la Unidad (AGG y BFB).

Las variables se han resumido mediante estadísticos descriptivos adecuados a la naturaleza de cada variable: medidas de tendencia central (media) y de dispersión (desviación estándar) para las variables cuantitativas $\mathrm{y}$ frecuencias absolutas y relativas para las cualitativas. La comparación entre las variables cuantitativas se ha llevado a cabo con la prueba t de Student cuando una de ellas fue cuantitativa. El nivel de error alfa o significación estadística escogido fue inferior a $5 \%$. Los cálculos estadísticos se realizaron con el paquete estadístico PASW 18,0 (SPSS Inc.).

El estudio fue aprobado por el Comité Ético de Investigación con Medicamentos de la Gerencia de Atención Integrada de Ciudad Real.

\section{RESULTADOS}

Durante 14 meses fueron atendidos 148 pacientes con NED con una edad media de $65,4 \pm 14,6$ años. $66,2 \%$ fueron varones y 33,8 \% mujeres. No hubo diferencias estadísticamente significativas entre las edades de ambos sexos. Teniendo en cuenta el tamaño poblacional del área de salud atendida por nuestra Unidad de Nutrición (y asumiendo pérdidas, probablemente escasas pero no cuantificadas, en el seguimiento ambulatorio por distintos motivos) la tasa de prevalencia estimada de pacientes con NED en nuestro entorno fue de 13 casos por 100.000 habitantes en el momento de finalizar la recolección de datos.

Las enfermedades más frecuentes que justificaron la NED fueron: los accidentes cerebrovasculares (16,9\%), el retraso psicomotor $(10,1 \%)$, la neoplasia de laringe $(8,1 \%)$ y la demencia vascular o Alzheimer.

La comparación entre las tres principales características de las fórmulas de nutrición enteral (contenido calórico, proteínas y fibra) pautadas se recogen en la Figura 1. Las fórmulas normoproteicas fueron en su mayoría utilizadas en pacientes con enfermedades neurológicas, degenerativas y vasculares, (66,6\%); mientras que las hiperproteicas fueron empleadas con mayor frecuencia en pacientes oncológicos (44,4\%). Los pacientes a quienes se indicó fórmulas nutricionales hiperproteicas fueron significativamente más jóvenes $(61,1$ frente a 68,2 años; $\mathrm{p}<0,05)$. Las características específicas de las tres más utilizadas fueron: normocalórica normoproteica con fibra (NC/NPCF), hipercalórica hiperproteica sin fibra (HC/HPSF), y, con el mismo número de pacientes utilizándolas hipercalórica normoproteica sin fibra (HC/NPSF) y normocalórica normoproteica sin fibra (NC/NPSF), con una distribución porcentual de $17,6 \%, 12,8 \%, 8,8 \%$, respectivamente. Solo 26 pacientes estaban con una fórmula específica, fundamentalmente para la diabetes (el $50 \%$ de todas las pautadas).

Respecto a las vías de acceso de la nutrición enteral, el $68 \%$ de los pacientes tenían un acceso enteral invasivo, fundamentalmente gastrostomías endoscópicas percutáneas (GEP). La Figura 2 muestra el número de pacientes con las distintas técnicas invasivas de colocación de sondas de nutrición. El $32 \%$ de los pacientes tenían un acceso enteral no invasivo: $24 \%$ sondas nasogástricas y $8 \%$ nasoyeyunales. No hubo diferencias significativas al comparar la media de edad de los pacientes con acceso invasivo y no invasivo. La Figura 3 recoge la utilización del tipo de acceso (invasivo o no) en las cuatro patologías prevalentes. $15 \%$ (23) de los pacientes portaban sondas pospilóricas (12 naasoyeyunales, 7 de yeyunostomías quirúrgicas y 4 gastroyeyunales). La causa más frecuente para la colocación de 


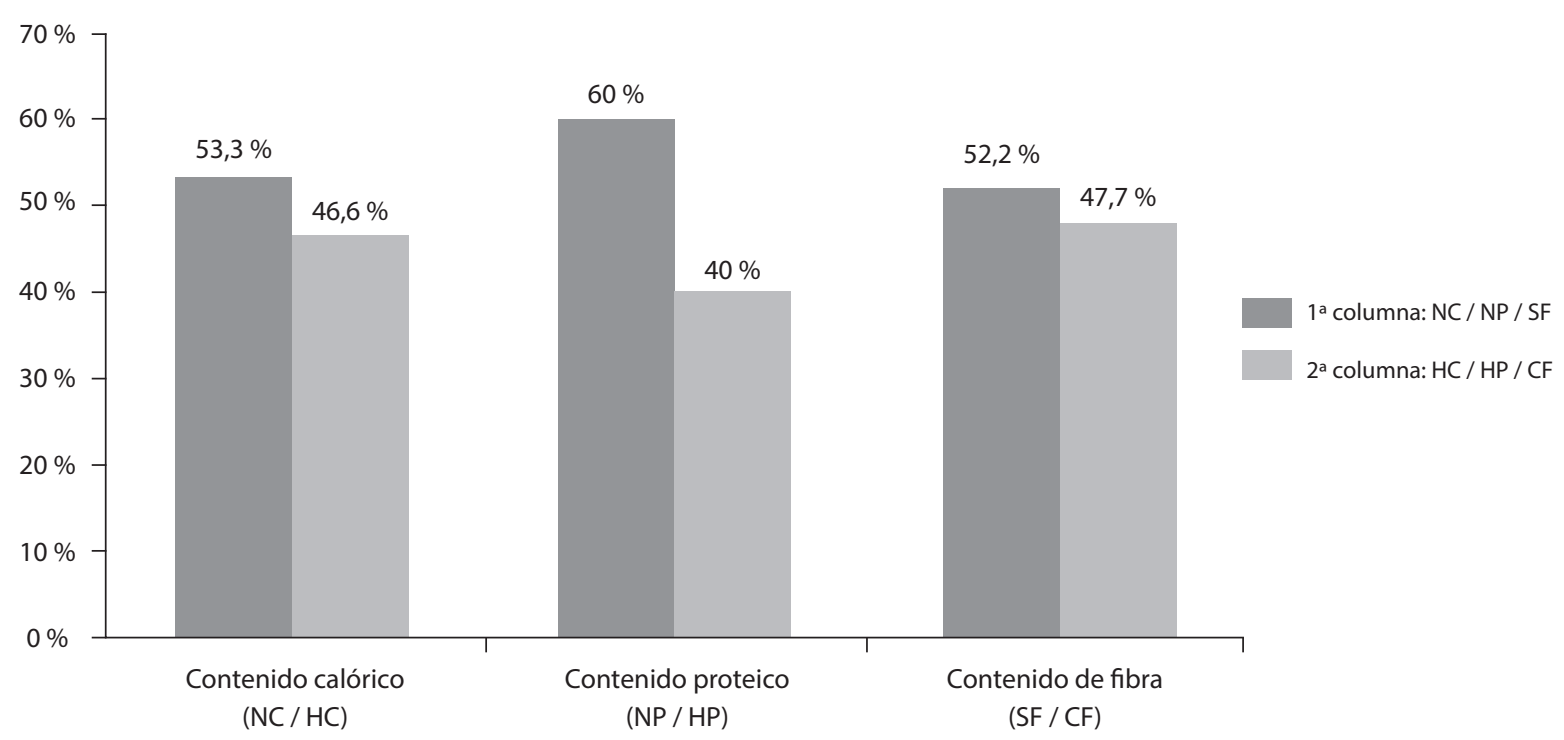

Figura 1. Características principales de las fórmulas nutricionales empleadas. 1a: columna: características de dietas estándar. 2a: columna: características de dietas no estándar. NC: normocalórica. HC: Hipercalórica. NP: Normoproteica. HP: Hiperproteica. SF: Sin fibra. CF: Con fibra.

las sondas entéricas fue la neoplasia gástrica, en 34,8 \% de los casos. Solo en 7,4 \% de los pacientes la forma de administración de la nutrición enteral fue en infusión continua - en $54,5 \%$ de estos pacientes las sondas de administración utilizadas fueron pospilóricas y en $45,4 \%$ de ellos la fórmula nutricional empleada fue oligomérica - en el resto la administración fue intermitente; sobre todo por gravedad.

El $57 \%$ de los pacientes presentaron complicaciones importantes, consideradas como tales las que obligaron a un ingreso hospitalario o las que resultaron en el fallecimiento del paciente. $26,4 \%$ de los pacientes del estudio fallecieron durante el seguimiento, todos tenían una vía de acceso invasiva. Las principales causas de muerte fueron la infección respiratoria, la falla multiorgánica y la sepsis $(43,58 \%, 25,64 \%$ y $5,1 \%$, respectivamente). La mortalidad fue más frecuente entre los pacientes que utilizaban accesos invasivos (GEP): la mortalidad más importante la presentaron los pacientes con gastrostomías endoscópicas percutáneas (GEP) con 33,8 $\%$ respecto al total de las sondas invasivas. No se presentaron fallecimientos en los 35 pacientes con sonda nasogástrica durante el periodo estudiado.

En 16,54 \% del total de los pacientes fue posible la retirada de la sonda de nutrición durante su seguimiento por reinicio de ingesta oral; de ellos, $56,5 \%$ portaba una sonda nasogástrica. De estos pacientes, 34,7 \% corres- pondió a pacientes que la precisaron tras intervenciones quirúrgicas de cabeza y cuello, oncológicas o no.

\section{DISCUSIÓN}

La NED ha permitido que muchos pacientes puedan volver a su domicilio con las ventajas que de ello se derivan para estos y sus familias, pero también para los Sistemas Nacionales de Salud por el alto coste asociado a la hospitalización ${ }^{(6)}$. No obstante, esta intervención terapéutica no está exenta de complicaciones por lo que los pacientes deben ser evaluados periódicamente de forma ambulatoria ${ }^{(8)}$. En este estudio se analizaron las características de los pacientes con NED de nuestra área de salud obtenidas de una consulta de enfermería, perteneciente a la unidad de nutrición clínica, específicamente diseñada para el seguimiento telefónico de estos. La mayor parte de los resultados fueron comparados con los obtenidos por el grupo de trabajo de la Sociedad Española de Nutrición Parenteral y Enteral (SENPE) con el registro NADYA (Nutrición Artificial Domiciliaria y Ambulatoria), un registro nacional en el que los profesionales pueden incluir los datos de sus pacientes de forma voluntaria y de los que se realizan evaluaciones periódicas.

La media de edad de nuestros pacientes fue de 65,4 años, sin diferencia entre ambos sexos; otros trabajos 


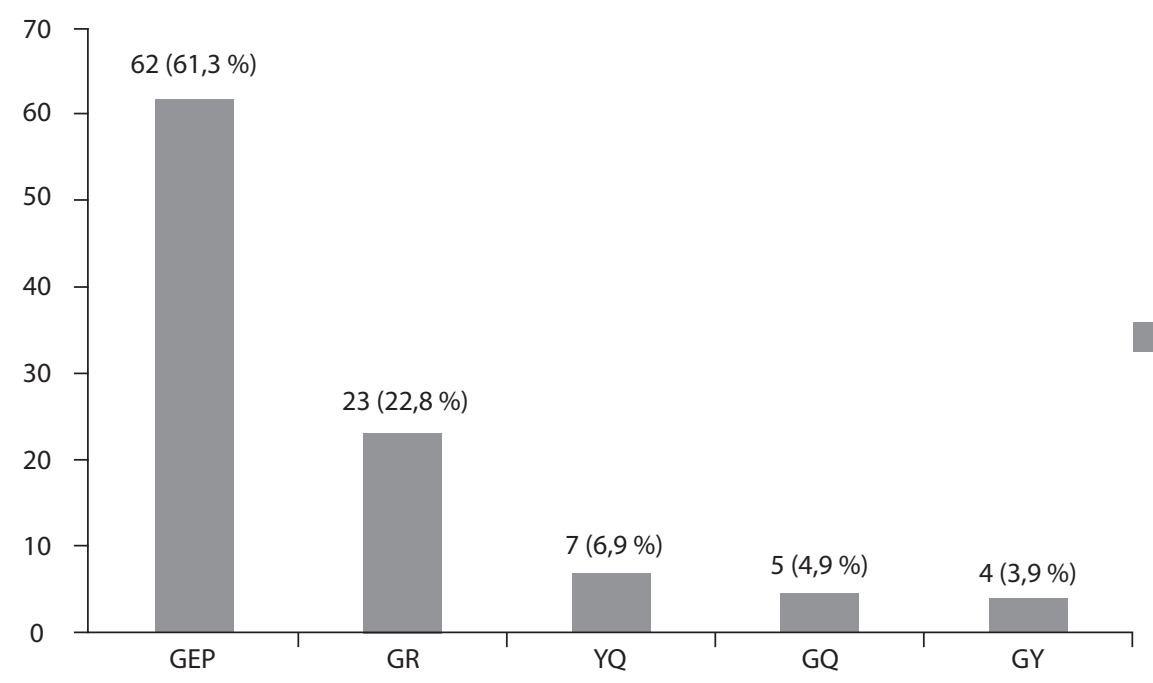

Figura 2. Distribución del número de pacientes, absoluto y porcentual, con las distintas técnicas invasivas de colocación de sondas de nutrición. GEP: Gastrostomía endoscópica percutánea; GR: Gastrostomías radioscópicas, YQ: Yeyunostomías quirúrgicas, GQ: Gastrostomías quirúrgicas, GY: Gastroyeyunostomías.

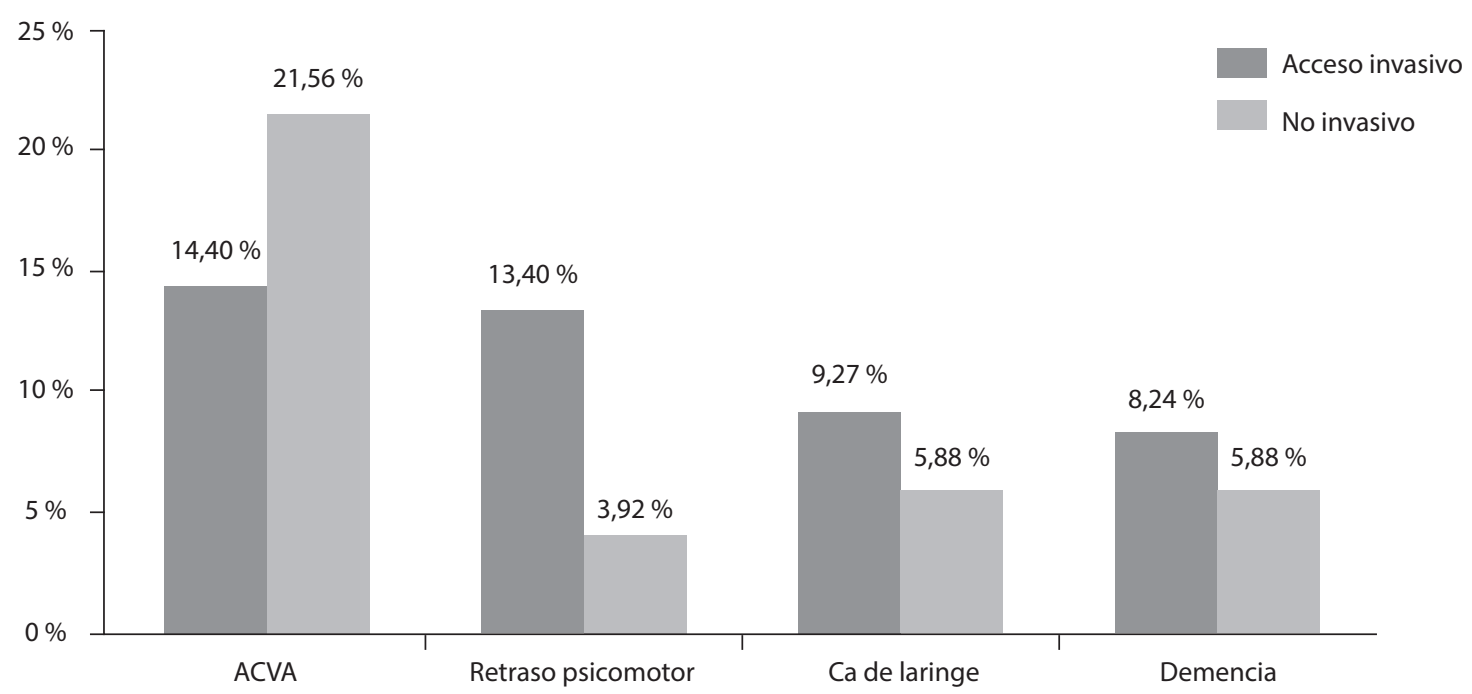

Figura 3. Tipo de sonda (invasivo o no) según las patologías más frecuentes.

publicados sí han encontrado una edad media mayor en las mujeres ${ }^{(4,10)}$. La relación entre varones y mujeres en nuestra muestra fue de 2:1 a diferencia de otros grupos que contabilizan una representación porcentual simi$\operatorname{lar}^{(4,5)}$. La prevalencia de esta técnica de nutrición, definida como el número de pacientes con NE activa (que continuaban en fecha final de la recogida de los datos), es, en nuestra muestra, más elevada que la publicada en el registro nacional español NADYA entre los años $2014-2017^{(4,10)}$. El hecho de que en este registro nacional se analizaran las prevalencias anuales y en nuestra muestra una población acumulada a lo largo de varios 
años hace que los resultados de esta comparación deban ser tomados con reserva.

La enfermedad neurológica (por diferentes causas) supone la principal indicación de prescripción de NED en nuestra población, al igual que ocurre a nivel global en España ${ }^{(4)}$.

En nuestro caso la mayor parte de los pacientes tenían una vía de acceso invasiva (principalmente PEG), a diferencia de los datos obtenidos en los distintos cortes del grupo NADYA en los que el acceso más frecuente fue la sonda nasogástrica ${ }^{(4,5,10)}$. Este mayor porcentaje de accesos invasivos respecto a los de los registros NADYA podría ser explicado por dos razones: una, porque el seguimiento continuo de nuestros pacientes permite actualizar las modificaciones en el tratamiento de estos, siendo los datos recogidos los relativos al último contacto telefónico, de hecho cuando se evaluó un subgrupo de pacientes del registro NADYA 2011-2012 con un segundo episodio de NED el porcentaje de estos con gastrostomías fue similar al nuestro $(63,5 \%)^{(5)}$ y dos, por características específicas de funcionamiento de la unidad de nutrición de nuestro hospital: en el área sanitaria prácticamente todos los pacientes con NE que han sido hospitalizados por cualquier motivo en cualquier unidad son valorados antes del alta hospitalaria por algún miembro de nuestro grupo de trabajo y si el tiempo estimado de la nutrición es más prolongado de 4 - 6 semanas se recomienda este tipo de acceso y se procede a su colocación durante el periodo de hospitalización, lo que sin duda ayuda a incrementar el número de pacientes con este; porque la colocación de estas sondas de forma ambulatoria, más demorada y con mayores trámites administrativos, lleva a los pacientes y cuidadores en muchos casos a desestimar estos procedimientos.

En cuanto a las complicaciones evolutivas de los pacientes con NED identificamos un menor porcentaje acumulado de fallecimientos $(26,4 \%)$ respecto a la media del registro nacional español (50 \%) y un porcentaje similar de pacientes en los que se logró recuperar la ingesta oral, aunque estos hallazgos deben ser interpretados con precaución porque el periodo analizado por el registro NADYA es mayor ( 2 años) que el nuestro (14 meses).

Respecto al tipo de fórmulas de nutrición utilizadas, llama la atención que, aunque la mayor parte de estas son normocalóricas y normoproteicas, el porcentaje de pacientes que consumía fórmulas hiperproteicas fue muy elevado (40\%). Que los pacientes que utilizaban estas fórmulas fueran más jóvenes y con patología oncológica apoya que se hace un uso de estas en pacientes con mayor catabolismo proteico, siguiendo las recomendaciones actuales de los grupos de expertos ${ }^{(11)}$, lo que también ocurre con la recomendación de fórmulas específicas y oligoméricas, empleada en un grupo de pacientes muy pequeño con unas características muy definidas en las que estas fórmulas han demostrado cierto beneficio sobre las estándar ${ }^{(12)}$.

Aunque nuestra muestra tiene un tamaño objetivamente razonable es claramente menor que el analizado en los distintos cortes del registro nacional español (NADYA) tomado como referencia, por lo que las comparaciones entre ambas deben ser valoradas con la prudencia debida; siendo ésta la principal limitación del estudio. Sin embargo, el tipo de seguimiento periódico tan frecuente ( 1 mes o menos), hace que los datos estén continuamente actualizados, lo que nos lleva a tener una imagen real constante de nuestro grupo de pacientes y garantiza la fiabilidad de los datos; que es sin duda una de las fortalezas del trabajo.

\section{CONCLUSIONES}

El presente estudio permitió conocer las características de los pacientes con NED del área de salud obtenidas de una consulta de enfermería, perteneciente a la Unidad de Nutrición Clínica del Hospital General Universitario de Ciudad Real. La enfermedad neurológica es la principal indicación de prescripción de la NED. La prevalencia del uso de NED en nuestra área es elevada, así como la utilización de vías de acceso invasivas. El papel de enfermería es muy importante en el control ambulatorio de los pacientes con NED.

\section{Financiación}

Los autores no han recibido ningún tipo de ayuda económica para llevar a cabo este estudio.

\section{Conflicto de intereses}

No existen.

\section{Declaración de autoría}

AGG fue responsable de la concepción, diseño del estudio y interpretación de los datos; MJHS realizó el diseño de la base de datos; AGG, MJHS, BFBP, CFV, PAMC, IDO participaron en la adquisición y recopilación de datos. IDO participó en la revisión bibliográfica. Todos los autores revisaron y aprobaron la versión final del manuscrito. 


\section{Referencias bibliográficas}

1. Mundi MS, Pattinson A, McMahon MT, Davidson J, Hurt RT. Prevalence of Home Parenteral and Enteral Nutrition in the United States. Nutr Clin Pract. 2017; 32(6): 799-805. doi: $10.1177 / 0884533617718472$.

2. Jukic PN, Gagliardi C, Fagnani D, Venturini C, Orlandoni P. Home Enteral Nutrition therapy: Difficulties, satisfactions and support needs of caregivers assisting older patients. Clin Nutr. 2017;36(4):1062-7. doi: 10.1016/j.clnu.2016.06.021.

3. Martin K, Gardner G. Home Enteral Nutrition: Updates, Trends and Challenges. Nutr Clin Pract. 2017; 32(6): 712-21. doi: $10.1177 / 0884533617701401$

4. Wanden-Berghe C, Campos C, Burgos R, Álvarez J, Frias L, Matia P, et al. Registro del Grupo NADYA-SENPE de Nutrición Enteral Domiciliaria en España; años 2016 y 2017. Nutr Hosp. 2019; 36(1):233-7. doi: 10.20960/nh.02365

5. Wanden-Berghe C, Matía Martín P, Luengo Pérez LM, Cuerda Compes C, Burgos Peláez R, Alvarez Hernández J, et al. Home enteral nutrition in Spain; NADYA registry 2011-2012. Nutr Hosp. 2014;29(6):1339-44. doi: 10.3305/nh.2014.29.6.7360.

6. Maeda M, Fukuda H, Shimizu S, Ishizaki T. A comparative analysis of treatment costs for home-based care and hospitalbased care in enteral nutrition patients: A retrospective analysis of claims data. Health Policy. 2019;123(4):367-72. doi: 10.1016/j.healthpol.2018.12.006.
7. Cederholm T, Barazzoni R, Austin P, Ballmer P, Biolo G, Bischoff SC, et al. ESPEN guidelines on definitions and terminology of clinical nutrition. Clin Nutr. 2017; 36(1):49-64. doi: 10.1016/j.clnu.2016.09.004.

8. Gobiernos de España. Ministerio de Sanidad y Consumo. Guía de nutrición enteral domiciliaria en el Sistema Nacional de Salud. Madrid: Ministerio de Sanidad y Consumo. 2008. [Internet]. [Fecha de consulta 30 de Enero de 2020]. Disponible en: https://www.mscbs.gob.es/profesionales/ prestacionesSanitarias/publicaciones/docs/guiaNED.pdf

9. Bischoff SC, Austin P, Boeykens K, Chourdakis M, Cuerda C, Jonkers-Schuitema C, et al. ESPEN guideline on home enteral nutrition. Clin Nutr. 2020;39(1):5-22 doi: 10.1016/j. clnu.2019.04.022

10. Wanden-Berghe C, Luengo LM, Álvarez J, Burgos R, Cuerda C, Matía P, et al. Registro del grupo NADYA-SENPE de nutrición enteral domiciliaria en España, años 2014 y 2015. Nutr Hosp. 2017; 34(1):15-18. doi: 10.20960/nh.970.

11. Alvarez HJ, Peláez TN y Muñoz JA. Utilización clínica de la Nutrición Enteral. Nutr. Hosp. 2006;21(Supl. 2):87-99.

12. Fernández PJI, Romero PJA, García Pérez-Sevillano F. Fórmulas de nutrición enteral: revisión de la evidencia actual. Nutr Clin Med. 2017;11(3):171-86. 